\title{
Estimation of longitudinal and lateral vehicle velocities: an algebraic approach
}

\author{
Jorge VILLAGRA*, Brigitte d'ANDRÉA-NOVEL*, Michel FLIESS ${ }^{\dagger}$, Hugues MOUNIER ${ }^{\dagger}$ \\ *Centre de Robotique, École des Mines de Paris \\ 60 boulevard Saint-Michel, 75272 Paris cedex 06, France \\ jvillagreing.uc3m.es \& brigitte.dandrea-novel@ensmp. fr \\ $\dagger$ INRIA-ALIEN \& LIX (CNRS, UMR 7161) \\ École polytechnique, 91128 Palaiseau, France \\ Michel.Fliessepolytechnique.edu \\ †Institut d'Électronique Fondamentale (CNRS, UMR 8622) \\ Université Paris-Sud, 91405 Orsay, France \\ Hugues.Mounier@ief.u-psud.fr
}

\begin{abstract}
This paper presents a new approach for estimating vehicle velocities at its gravity center. The proposed strategy relies on recent algebraic techniques for numerical differentiation and diagnosis. We do not use any tire model in order to obtain an estimation, which is robust with respect to model uncertainties (friction, ....). All available measurements in a mass-production car are however exploited.
\end{abstract}

\section{INTRODUCTION}

\section{A. Generalities}

Active security systems like ESP, lane keeping, ABS or even Stop\&Go strongly depend on tire/road interaction forces. A good knowledge of the vehicle velocities at the center of gravity is also very important. Hence several tire model $^{1}$ based estimation methods have been developed in recent years.

A single track model, linear or not according to the tire models, has been often employed (see [26] and the references therein). In [3] and [21], the authors propose different versions of the extended Kalman filter. A $H_{\infty}$ filter is suggested in [16]; [15] proposes a neural network approach and [6] a recursive least squares method; [23] uses sliding modes techniques; [2] implements a high gain observer; [4], [5], [10], [11], [12], [13], [24], [25] exploit a Lyapunov function in order to synthesize asymptotic nonlinear observers of tire forces or vehicle velocities. Those approaches are only valid under very favorable conditions, such as small steering angles or slowly variable velocities. Thus, in many situations they are extremely sensitive to friction conditions. Some other authors (see, e.g., [18], [20]) have tried to incorporate some kind of cornering stiffness variability using relaxation models, where parameters are identified online. In all those methods, tire models are hard to exploit because their parameters are too numerous and/or not easily identifiable. We have therefore preferred to use simple dynamical models,

\footnotetext{
${ }^{1}$ See, e.g., [13], [19] and the references therein for details on vehicle dynamics.
}

with a minimal number of parameters, and to exploit at best the information coming from vehicle's sensors.

The wheel rotation obviously satisfies

$$
I_{r} \dot{\omega}=-r F_{x}+C
$$

where $I_{r}$ and $r$ are, respectively, the inertia momentum and the wheel radius, $\omega$ is the wheel rotation speed, $F_{x}$ is the longitudinal tire force at ground/tire contact point, and $C$ is the motor/brake torque. Since $\omega$ can be measured, $I_{r}$ and $r$ values can be approximately known, and if we suppose that $C$ can be somehow estimated, Eq. (1) provides a first estimation of $F_{x}$. Besides, a good estimation of $F_{z}$ can be obtained (see, e.g., [22]), so that an acceptable friction estimator could be synthesized. However, such an estimator would not be very robust with respect to imprecisions on torque estimation and wheel radius value. Thus, improving the estimation requires a good knowledge of the vehicle longitudinal velocity. Therefore, obtaining velocities on the center of gravity is the first step towards reliable vehicle onboard control laws and diagnosis.

Besides, slip ratio knowledge requires a precise estimation of longitudinal velocity, which is obtained by expressing longitudinal acceleration with respect to a rotating frame:

$$
\left\{\begin{array}{l}
\gamma_{x}(t)=\dot{V}_{x}(t)-\dot{\psi}(t) V_{y}(t) \\
\gamma_{y}(t)=\dot{V}_{y}(t)+\dot{\psi}(t) V_{x}(t)
\end{array}\right.
$$

where $V_{y}$ is the lateral velocity, $\dot{\psi}$ the yaw rate, $\gamma_{x}$ the longitudinal acceleration and $\gamma_{y}$ the lateral acceleration. Even if vertical, pitch and roll dynamics are neglected in this model, it remains very realistic in most situations. Furthermore, it is completely independent of the considered vehicle, and therefore on its parameters. No parameter is then required; only the three previous measurements (plus odometer informations) will be necessary. Let us note that, contrarily to most of the previous approaches, we will not use the steering angle input, usually accessible on the CAN bus. 


\section{B. Outline of the article}

Section II presents the general approach in order to estimate longitudinal and lateral velocities. Some tools from diagnosis will be introduced and we shall explain how to tackle the unobservability problems associated to Eq. (2). In a real automotive context, low cost sensors are generally used, so that measurements are usually highly noise-corrupted. Filtering these signals is the object of Section III, where a summary on numerical differentiation is presented. Section IV is devoted to a precise explanation of the implemented estimation algorithms. Section $\mathrm{V}$ provides convincing numerical results of very realistic simulations. The conclusion in Section VI is sketching some future works.

\section{Diagnosis For Velocities ESTIMATION}

As previously mentioned, Eq. (2) and the available measurements of $\gamma_{x}, \gamma_{y}$ and $\dot{\psi}$ on high-end cars will be used to precisely estimate $V_{x}$ and $V_{y}$. The next proposition shows the mathematical impossibility of estimating simultaneously $V_{x}$ and $V_{y}$ from the previous three measurements ${ }^{2}$.

Proposition 1: Longitudinal and lateral velocities $\left(V_{x}, V_{y}\right)$ cannot be simultaneously estimated from equations:

$$
\begin{aligned}
& \gamma_{x}(t)=\dot{V}_{x}(t)-\dot{\psi}(t) V_{y}(t) \\
& \gamma_{y}(t)=\dot{V}_{y}(t)+\dot{\psi}(t) V_{x}(t)
\end{aligned}
$$

except if values $V_{x_{t_{0}}}$ and $V_{y_{t_{0}}}$ at initial time $t_{0}$ are known.

Proof: Eq. (2) can be rewritten as a single equation in the complex domain if the linear combination (3)+i(4), where $i=\sqrt{-1}$, is considered:

$$
\dot{V}_{x}(t)+i \dot{V}_{y}(t)=\gamma_{x}(t)+i \gamma_{y}(t)+\dot{\psi}\left(-i V_{x}(t)+V_{y}(t)\right)
$$

which is equivalent to the complex differential equation

$$
\dot{V}=-i \dot{\psi}(t) V(t)+\gamma(t), V\left(t_{0}\right)=V_{0}
$$

where $V(t)=V_{x}(t)+i V_{y}(t)$ and $\gamma(t)=\gamma_{x}(t)+i \gamma_{y}(t)$. Eq. (5) has a unique solution if, and only if, the initial condition $V_{0}=V_{x}\left(t_{0}\right)+i V_{y}\left(t_{0}\right)$ is known.

It hardly seems possible to have good estimators of longitudinal and lateral velocities using only Eq. (2). Indeed, a good estimation would require the precise knowledge of initial conditions at every sample (in order to avoid increasing offsets due to integration).

Our strategy allows to use at best Eq. (2) by means of diagnosis tools (see, e.g., [9] and the references therein). Let us consider the velocities $(V x, V y)$ as the sum of an ideal term $(R x, R y)$ and a "disturbing" one ${ }^{3}(G x, G y)$

$$
\left\{\begin{array}{l}
V_{x}(t)=R_{x}(t)+G_{x}(t) \\
V_{y}(t)=R_{y}(t)+G_{y}(t)
\end{array}\right.
$$

where

- $R_{x}=r \omega_{t}$,

\footnotetext{
${ }^{2}$ This property may be related to a lack of observability (see [9] and the references therein).

${ }^{3}$ Those disturbing terms are nothing else than "poorly known" effects. See [8] for the control of poorly known systems.
}

- $r$ is the static wheel radius,

- $\omega_{t}=\frac{1}{4} \sum_{i=1}^{4} \omega_{i}$ is the mean rotation speed of the four wheels,

- $R_{y}=-L_{1} \dot{\psi}$,

- $L_{1}$ is the vehicle front wheelbase.

Remark 1: The difference between faultless and faulty behavior appears in a quite natural way in longitudinal dynamics. Concerning lateral dynamics, sideslip angle expression on the front axle's center $\beta_{1}$ can be used to obtain an equation similar to Eq. (6):

$$
\beta_{1}=\arctan \left(\frac{V_{y}+L_{1} \dot{\psi}}{V_{x}}\right) \Rightarrow V_{y}=-L_{1} \dot{\psi}+V_{x} \tan \left(\beta_{1}\right) .
$$

Differentiating both sides of Eq. (6) and replacing $\dot{V}_{x}$ and $\dot{V}_{y}$ by their corresponding expressions in Eq. (2) yields:

$$
\begin{aligned}
& \dot{R}_{x}=\dot{\psi} R_{y}-\dot{G}_{x}+\dot{\psi} G_{y}+\gamma_{x} \\
& \dot{R}_{y}=-\dot{\psi} R_{x}-\dot{G}_{y}-\dot{\psi} G_{x}+\gamma_{y}
\end{aligned}
$$

It yields for $G_{x}$ and $G_{y}$ :

$$
\begin{aligned}
& \dot{G}_{x}(t)=\dot{\psi}(t) G_{y}(t)-L_{1} \dot{\psi}^{2}(t)-r \dot{\omega}_{t}(t)+\gamma_{x}(t) \\
& \dot{G}_{y}(t)=-\dot{\psi}(t) G_{x}(t)-\dot{\psi}(t) r \omega_{t}(t)+L_{1} \ddot{\psi}(t)+\gamma_{y}(t)
\end{aligned}
$$

and if we consider initial conditions

$$
G_{x}\left(t_{0}\right)=0, G_{y}\left(t_{0}\right)=0
$$

then the integration of this system provides the necessary information about the instants where the assumption $V_{x}=r \omega_{t}$ is no more valid. If values coming from this numerical integration were precise, they could be sufficient to get a good estimation of longitudinal velocity (details will be presented in Section IV). However, highly corrupted signals provided by the vehicle sensors (especially accelerometers, see Fig. 1) and fixed integration step determined by signals sampling rate impose a signal pre-treatment. In addition, robust and real-time efficient numerical differentiators are also needed to render this approach feasible. It will be implemented on one hand to filter $\gamma_{x}, \gamma_{y}, \psi$, and on the other hand to estimate the derivatives $\dot{\omega}$ and $\ddot{\psi}$.

\section{Algebraic NUMERICAL DifFERENTIATION ${ }^{4}$}

Replace the convergent Taylor expansion $x(t)=\sum_{n \geq 0} a_{n} \frac{t^{n}}{n^{n}}$, $a_{n} \in \mathbb{C}$, by its truncated Taylor expansion $x_{N}(t)=\sum_{n=0}^{N} a_{n} \frac{t^{t}}{n !}$. It reads in the operational domain as (see, e.g., [27]):

$$
s^{N+1} x_{N}-s^{N} x_{N}(0)-s^{N-1} \dot{x}_{N}(0) \ldots-x_{N}^{(N)}(0)=0
$$

The derivatives at the origin $t=0$ are directly obtained from the linear system of equations

$$
\begin{array}{r}
s^{-v} \frac{d^{m}}{d s^{m}}\left\{x_{N}^{(N)}(0)+x_{N}^{(N-1)}(0) s+\ldots+x_{N}(0) s^{N}\right\}= \\
s^{-v} \frac{d^{m}}{d s^{m}}\left\{s^{N+1} x_{N}\right\}
\end{array}
$$

${ }^{4}$ See [9] for more details and various application to nonlinear systems, such as state and parameter estimation, diagnosis, and perturbation attenuation. Let us add moreover that the engineering literature on numerical differentiation is a huge one; see, e.g., the references in [9]. 
$m=0, \ldots, N, v \geqslant N+1$. This system is a triangular one with non-zero diagonal elements: it implies that the parameters $x_{N}^{(i)}(0)$, and therefore the coefficients $a_{0}, \ldots, a_{N}$ are linearly identifiable. Replace $x_{N}$ by $x$ in Eq. (8) to obtain the operational estimator $\left[x^{(i)}(0)\right]_{e_{N}}$ of $x^{(i)}(0)$.

Write the numerical estimates by expressing Eq. (8) back in the time domain, using the classical rules of operational calculus [27]:

- $\frac{c}{s^{\alpha}}, \alpha \geq 1, c \in \mathbb{C}$, by $c \frac{t^{\alpha-1}}{(\alpha-1) !}, t \geq 0$;

- $\frac{1}{s^{\alpha}} \frac{d^{n} x}{d s^{n}}$ by the iterated integral of order $\alpha$

$$
\begin{array}{r}
\int_{0}^{t} \int_{0}^{t_{\alpha-1}} \cdots \int_{0}^{t_{1}}(-1)^{n} \tau^{n} x(\tau) d t_{\alpha-1} \cdots d t_{1} d \tau= \\
\frac{(-1)^{n}}{(\alpha-1) !} \int_{0}^{t}(t-\tau)^{\alpha-1} \tau^{n} x(\tau) d \tau
\end{array}
$$

Remark 2: Iterated integrals have an averaging effect, and therefore act as low-pass filters, which attenuate noises, when viewed as highly fluctuating phenomena ${ }^{5}$.

Remark 3: A feasible real-time implementation is achieved thanks to a short time window.

A general expression of the $n^{\text {th }}$-derivative estimators can be written, in a window of size T, as follows [14]:

$$
\mathscr{P}_{v}(T)\left[\begin{array}{c}
x_{N}(0) \\
\dot{x}_{N}(0) \\
\vdots \\
x_{N}^{(N)}
\end{array}\right]=\int_{0}^{T} \mathscr{Q}_{v}(\tau) y(\tau) d \tau
$$

where the elements of the triangular matrix $\mathscr{P}_{V}(T)$ are, for $i=0, \ldots, N, j=0, \ldots, N-i$ :

$$
\left\{\mathscr{P}_{v}(T)\right\}_{i j}=\frac{(N-j) !}{(N-1-j) !} \frac{T^{\nu-N+i+j-1}}{(v-N+i+j-1)}
$$

The elements of the integral term are

$$
\left\{\mathscr{Q}_{v}(\tau)\right\}_{i}=\sum_{l=0}^{i} q_{i, l}(T-\tau)^{v-N-1-l} \tau^{i-l}
$$

with

$$
q_{i, l}=\left(\begin{array}{c}
i \\
l
\end{array}\right) \frac{(N+1) !}{(N+1-l) !} \frac{(-1)^{i-l}}{(v-N-1-l) !}
$$

Let us show as an example the particular expression of Eq. (10) for filtering the longitudinal acceleration and differentiating the yaw rate.

In both cases, the signal can be locally approximated by polynomials of degree 1 , i.e., $N=1$. Thus, $\gamma_{y}(t)=a_{0}+$ $a_{1} t, t \geqslant 0, a_{0}, a_{1} \in \mathbb{R}$, and $\dot{\psi}(t)=b_{0}+b_{1} t, t \geqslant 0, b_{0}, b_{1} \in \mathbb{R}$. It yields, if we take for instance $v=2$, the following estimators:

$$
\begin{aligned}
& \hat{\gamma}_{y}=\hat{a}_{0}=\frac{2}{T^{2}} \int_{0}^{T}(2 T-3 \tau) \gamma_{y}(\tau) d \tau \\
& \hat{\dot{\psi}}=\hat{b}_{1}=\frac{-3 !}{T^{3}} \int_{0}^{T}(T-2 \tau) \dot{\psi}(\tau) d \tau
\end{aligned}
$$

Observe in Fig. 1 that the noise attenuation is obviously related to the size of the estimation window.

\footnotetext{
${ }^{5}$ See [7] for details.
}
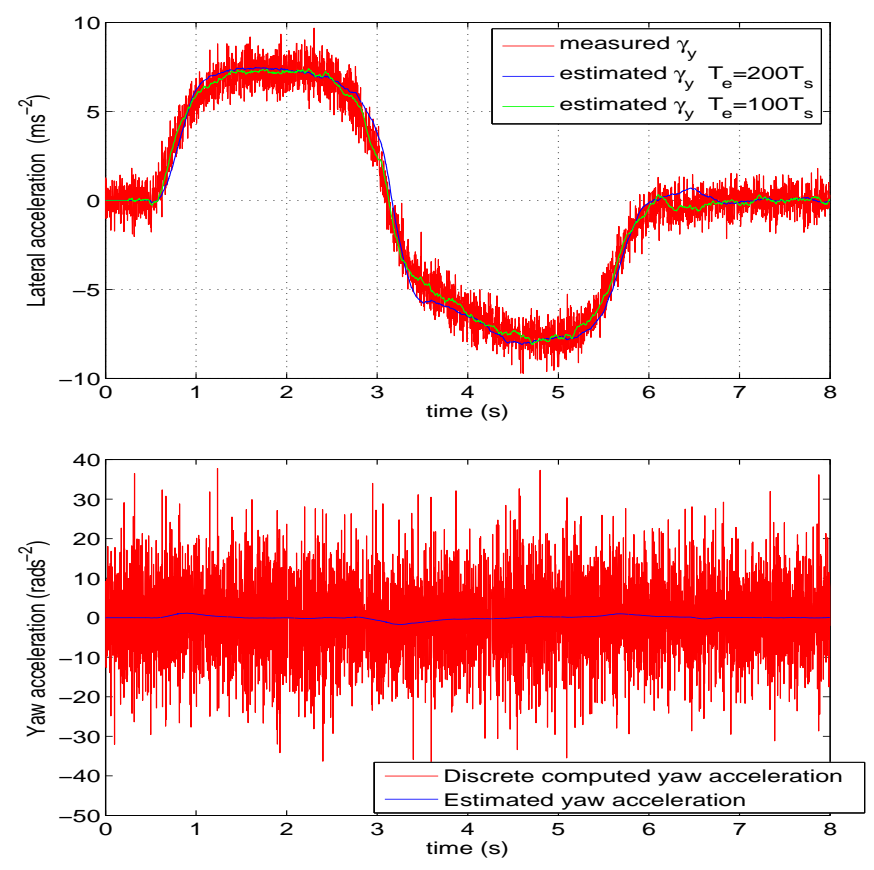

Fig. 1. Lateral acceleration filtering with 2 different window sizes (top). Comparison between a standard numerical differentiation of $\dot{\psi}$ and the one obtained with the algebraic methods (bottom)

\section{ESTIMATION ALGORITHMS}

From Eq. (2) and (7) the estimates of $V_{x}$ and $V_{y}$ can be obtained via the algorithms 1 and 2 .

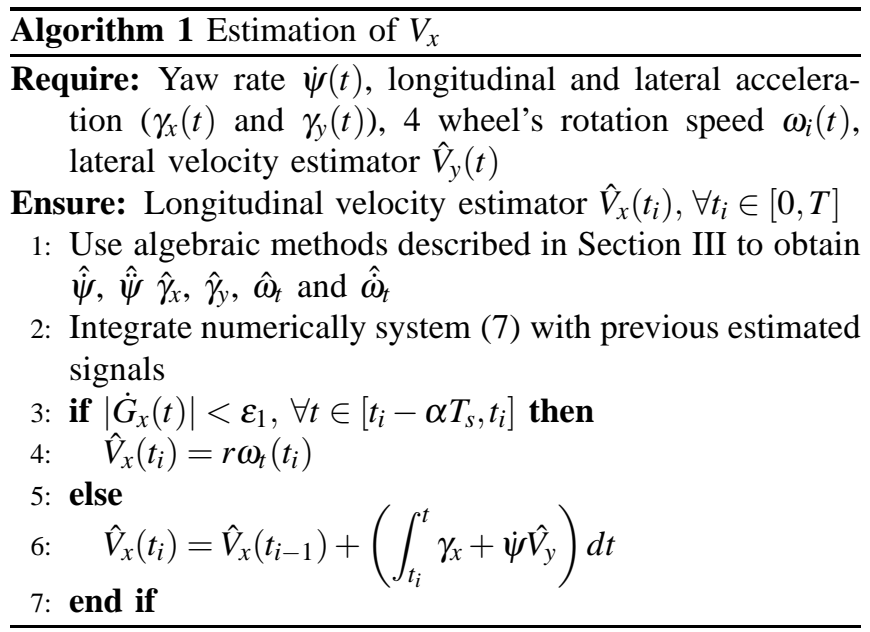

We see at the bottom of Fig. 2 that the obtained estimation is excellent and differs significantly from the crude estimate $r \omega_{t}$. Indeed, when slipping becomes significant, the algorithm does not rely on wheel speed (line 4 of algorithm 1), but also on accelerometer's measure (line 6 of algorithm 1). The switch between these two modes, bearing some resemblance with diagnosis techniques, relies on the significance of some residual related terms.

Note that these switching conditions (line 3 in algorithms $1 \& 2$ ) between integration modes are not solely obtained 


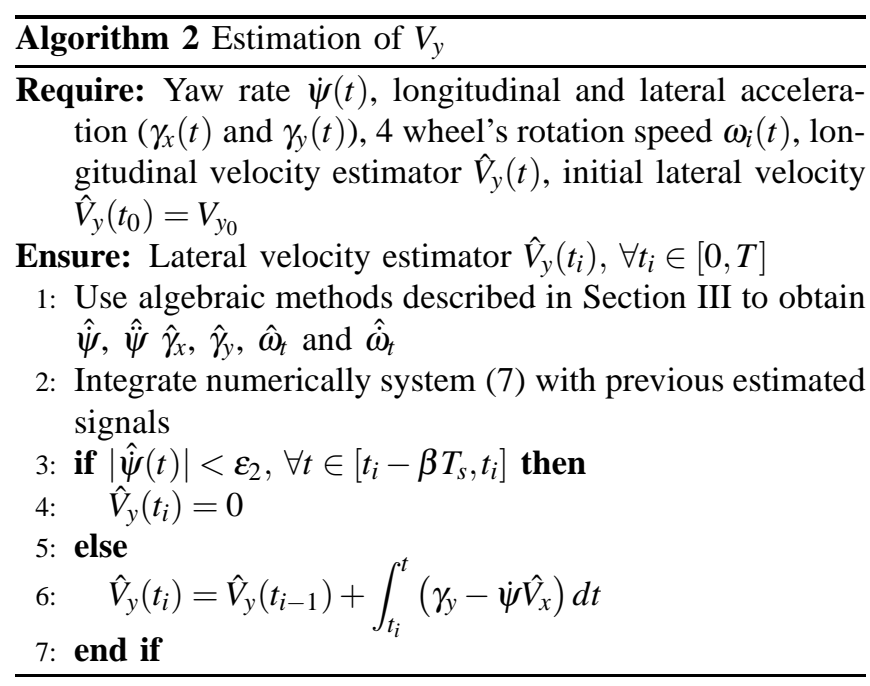

from $G_{x}$ and $G_{y}$ values. Hence, remark in Fig. 2 that, even if the evolution of $G_{x}$ is naturally linked to the validity of equation $V_{x}=r \omega_{t}, G_{x}$ does not stabilize around the origin for time instants $t \geqslant 7 \mathrm{~s}$. This phenomenon can be explained by two facts: measurements are highly corrupted by noise and integration step is fixed and constrained by the sampling rate.

$\dot{G}_{x}$ has been chosen as the switching variable in line 3 of algorithm 1 because, even if it is slightly noisy, it remains close to zero when $V_{x}=r \omega_{t}$. Note that the threshold condition established in algorithms 1 and 2 must be fulfilled all over a window of a user-predefined size $\alpha T_{s}$. The aim of this kind of constraint is to avoid sudden changes in estimation modes (line 4, resp. 5, in the algorithms 1 \& 2) each time the noisy $\dot{G}_{x}$ passes through zero. However, we see in Fig. 2 (middle graph, final zone) that the response time to stabilization around $\dot{G}_{x}=0$ is quite significant ${ }^{6}$.

The previous analysis concerning the switching variable for longitudinal dynamics is not valid anymore for lateral dynamics. Note in Fig. 3 that $\dot{G}_{y}$ is much more noisy than $\dot{G}_{x}$. This fact is probably due to remanent noise on yaw rate derivative estimation. Therefore, it seems much more convenient to use a noiseless estimate of the yaw rate to decide whether the lateral velocity is zero or if it is given by Eq. (4).

Remark 4: If the working horizon is quite long, it is preferable to reset Eq. (7) when the derivatives of the residuals $R_{x}$ and $R_{y}$ get close to zero.

\section{NUMERICAL EXPERIMENTS}

Our scenario (see steering angle and motor/brake torque in Fig. 4) leads to maximal longitudinal and lateral accelerations $\gamma_{x}$ and $\gamma_{y}$ which are approximatively equal to $0.8 g$ (see the last graph in Fig. 4).

Our numerical experiments are very realistic. We are employing a simulator of a vehicle with 14 degrees of freedom, and with complete drive-train and tire models (see [17]). The white Gaussian noises, which have been added

\footnotetext{
${ }^{6} \mathrm{~A}$ good trade-off between noisy or reactive estimation will be the engineers task.
}
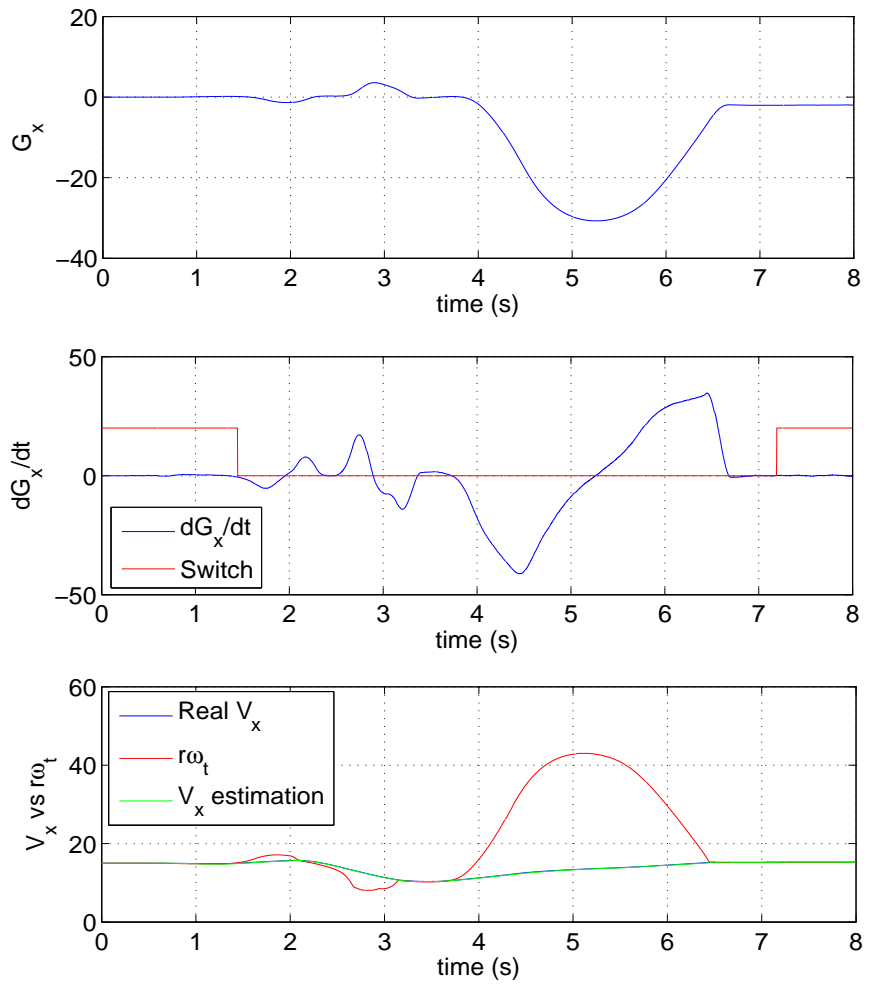

Fig. 2. $G_{x}(t)$ (top); $\dot{G}_{x}(t)$ and switching variable on longitudinal velocity estimation (middle); real and estimated longitudinal velocities and $r \omega_{t}$ (bottom)
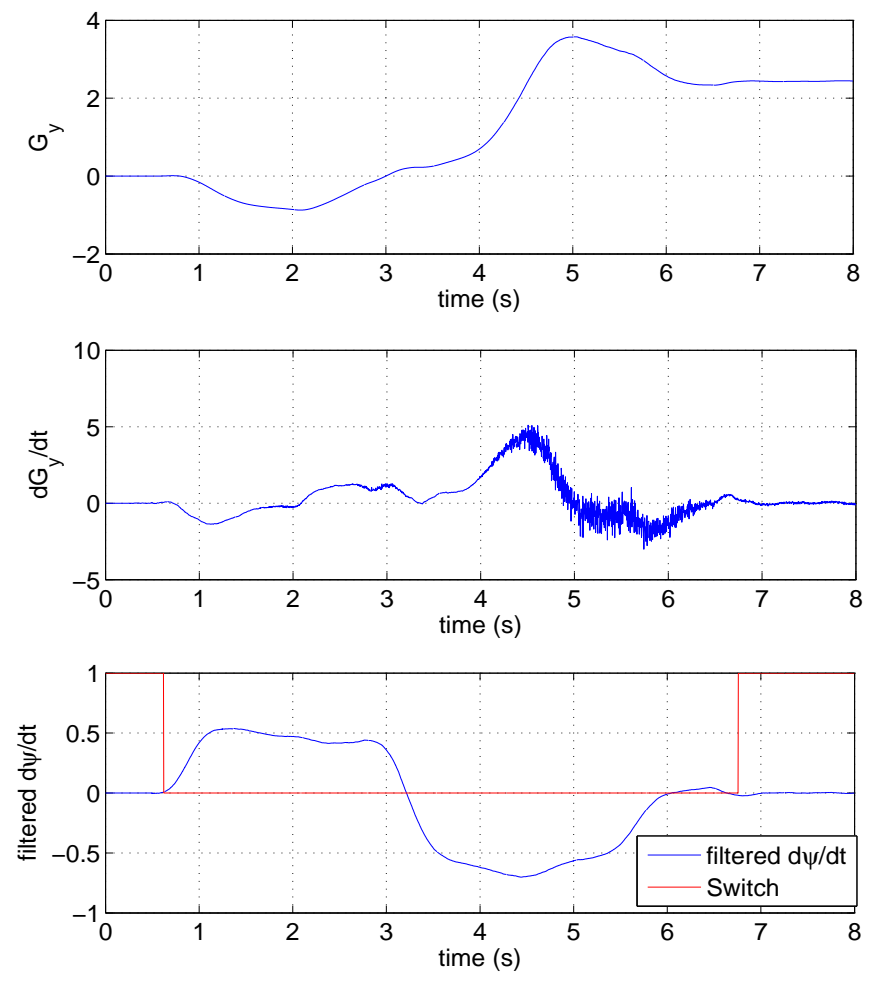

Fig. 3. $G_{y}(t)$ (top), $\dot{G}_{y}(t)$ (middle), and filtered $\dot{\psi}$ and switching variable on lateral velocity estimation (bottom) 

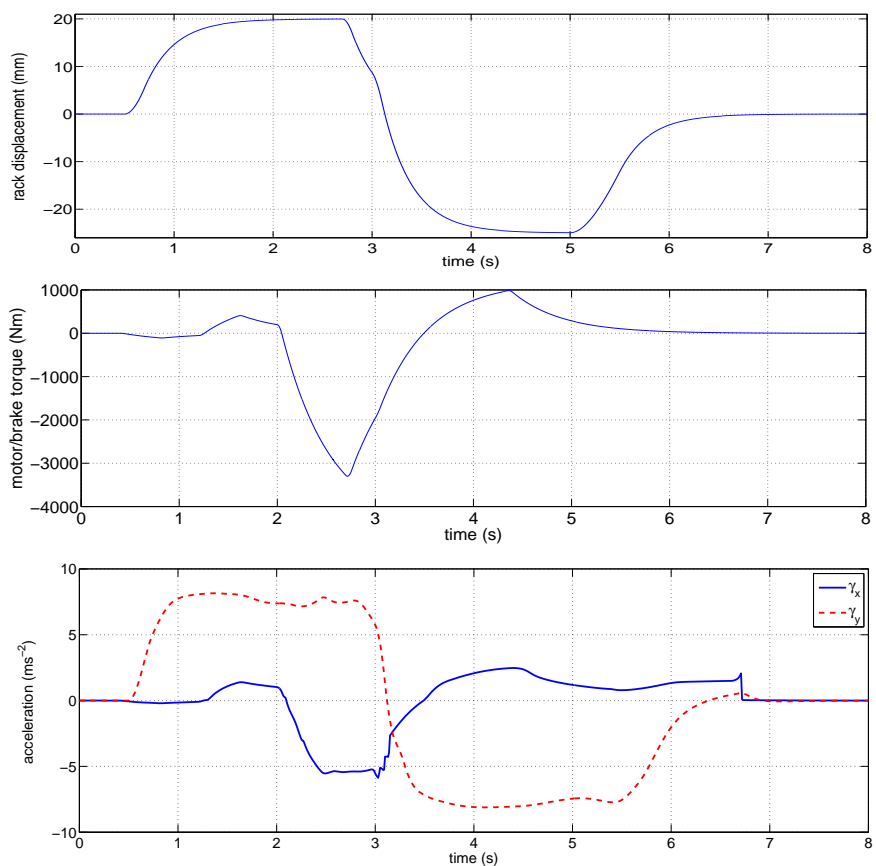

Fig. 4. Steering angle (rack displacement), motor/brake torque, and longitudinal/lateral accelerations of the vehicle.

to every measured signal, are stronger than those corrupting real onboard sensors. All necessary parameters to implement the previous algorithms are detailed in table I.

\begin{tabular}{|c|c|}
\hline Parameter & Value \\
\hline$T_{s}$ & $0.0025 \mathrm{~s}$ \\
$\varepsilon_{1}$ & 0.01 \\
$\varepsilon_{2}$ & 0.01 \\
$\alpha$ & 200 \\
$\beta$ & 100 \\
$T_{e}$ & 200 \\
\hline
\end{tabular}

TABLE I

PARAMETER VALUES

Fig. 5 shows the simulation results on a quite normal road, where the friction coefficient is $\mu=0.7$. The estimators perform pretty well. Fig. 6 and 7 show their behaviors when the driver actions are identical, but the friction coefficient is, respectively, higher $(\mu=0.9)$ and lower $(\mu=0.5)$.

Remark that the global behavior is very similar to the previous case. Thus, longitudinal velocity is rather well estimated and lateral velocity respects the trends previously shown. Nevertheless, note that estimation errors on $V_{x}$ have a larger influence on $V_{y}$ estimation than conversely.

\section{CONCLUSION}

A new estimation approach for vehicle velocities at its center of gravity is proposed. It is based on algebraic estimation techniques and diagnosis tools. A new strategy is presented, where only acceleration equations with respect to a rotating frame is used. Since this system of equations does
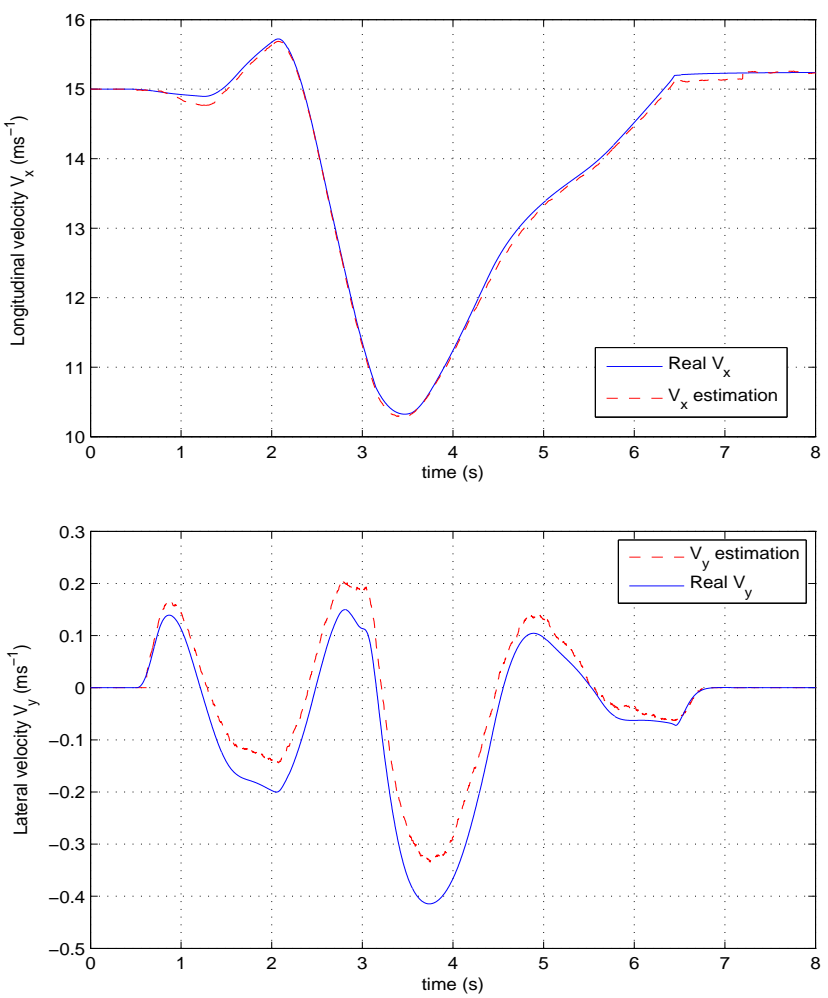

Fig. 5. Real and estimated longitudinal (top) and lateral (bottom) velocities with a friction coefficient $\mu=0.7$
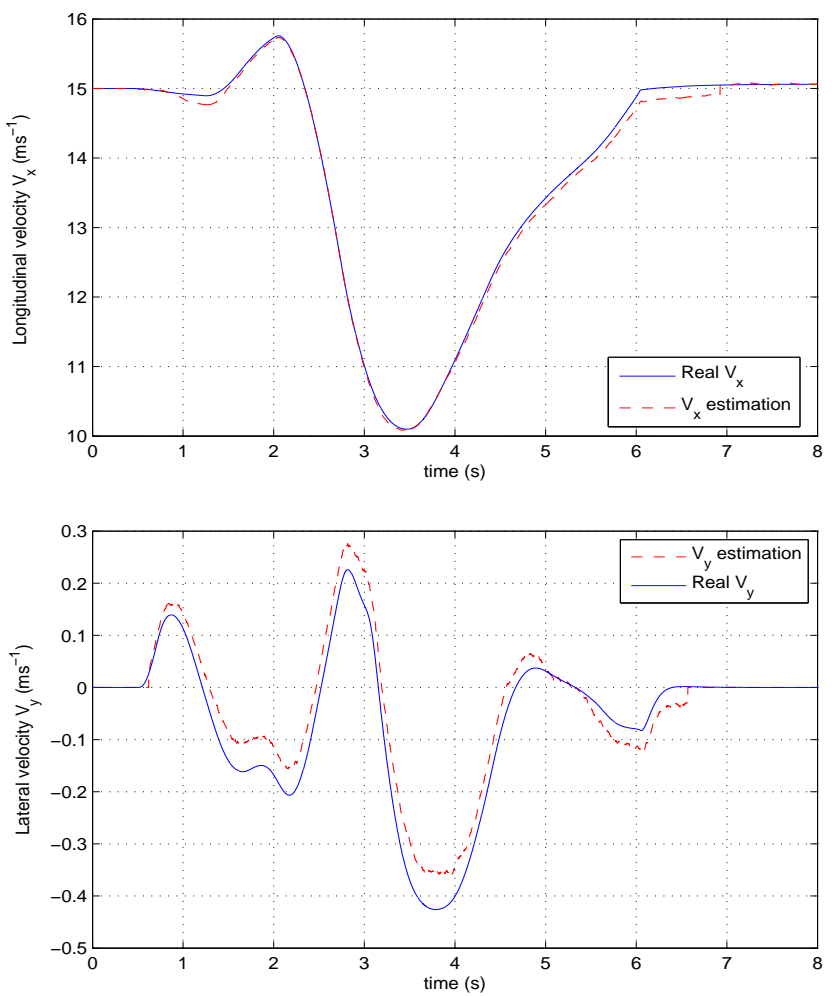

Fig. 6. Real and estimated longitudinal (top) and lateral (bottom) velocities with a friction coefficient $\mu=0.9$ 

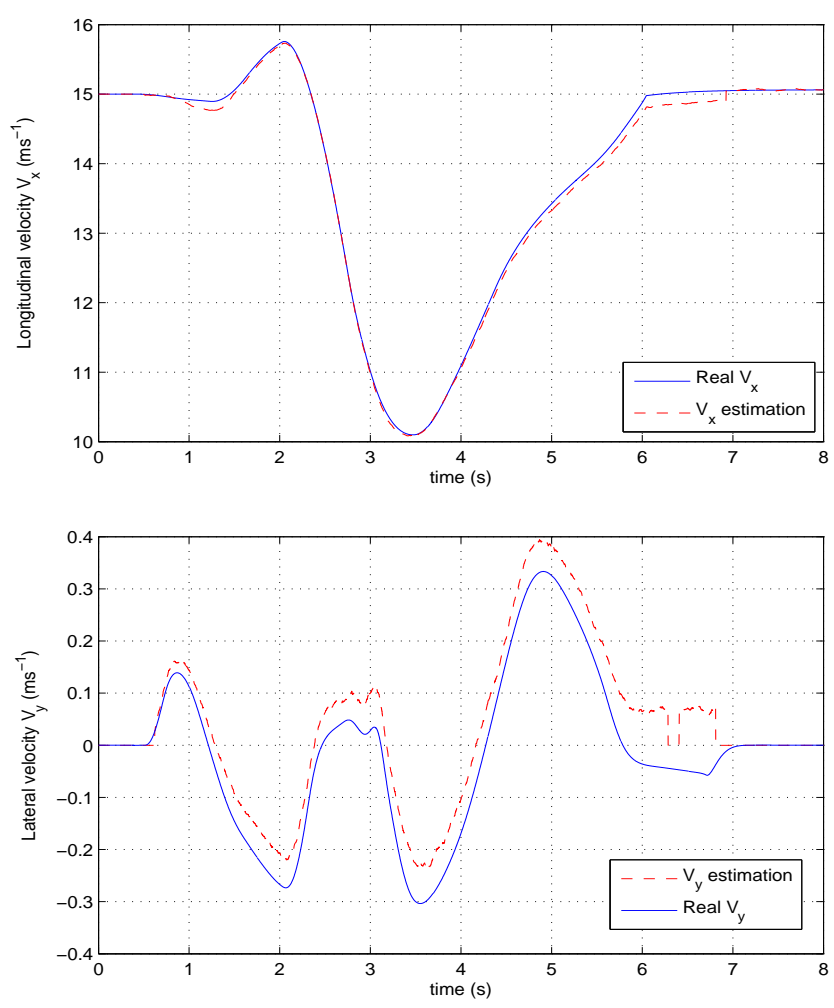

Fig. 7. Real and estimated longitudinal (top) and lateral (bottom) velocities with a friction coefficient $\mu=0.5$

not contain any vehicle parameter, the obtained estimators are remarkably robust.

Our first results not only exhibit excellent tracking quality, but also demonstrate good robustness with respect to noisy measurements and to road friction coefficient variations. Even if our simulations are accurately reproducing realistic situations, an experimental validation on a large range of scenarios (including banked roads) is necessary, and is under development on a prototype vehicle. Besides, similar techniques will be tested on tire forces and friction coefficient estimation.

See [1] for a preliminary application of our algebraic estimation techniques to traffic control.

Acknowledgments: This work was partially supported by the project Méthodes algébriques pour l'estimation temps réel : le cas des coefficients d'adhérence des efforts pneumatiques of the French GdR MACS (CNRS). The authors want also to express their gratitude to C. Join (INRIA-ALIEN \& CRAN, CNRS - NancyUniversité) and M. Mboup (INRIA-ALIEN \& Université RenéDescartes (Paris V)) for helping them to implement the numerical differentiators.

\section{REFERENCES}

[1] H. Abouaïssa, M. Fliess, C. Join, "Fast parametric estimation for macroscopic traffic flow model", Proc. $17^{\text {th }}$ IFAC World Congress, Seoul, 2008 (online http://hal.inria.fr/inria-00259032/en/).
[2] B. d'Andréa-Novel, H. Chou, J.-M. Coron, M. Ellouze, E. Fenaux, M. Pengov, F. Zarka, "An optimal control methodology for braking in corner with stability", Europ. Control Conf., Porto, 2001.

[3] G. Baffet, A. Charara, J. Stéphant, "Sideslip angle, lateral tire force and road friction estimation in simulations and experiments", Proc. IEEE Internat. Conf. Control Appl., Munich, pp. 903-908, 2006.

[4] M. Börner, R. Isermann, "Adaptive one-track model for critical lateral driving situations", Proc. Internat. Symp. Advanced Vehicle Control, Hiroshima, 2002.

[5] H. Cherouat, M. Braci, S. Diop, "Vehicle velocity, side slip angles and yaw rate estimation", Proc. IEEE Internat. Symp. Industrial Electronics, Dubrovnik, pp. 349-354, 2005.

[6] W. Deng, H. Zhang, "RLS-Based online estimation on vehicle linear sideslip", Proc. Amer. Control Conf., Minneapolis, 2006.

[7] M. Fliess, "Analyse non standard du bruit", C.R Acad. Sc. Paris, ser. I, vol. 342, pp. 797-802, 2006.

[8] M. Fliess, C. Join, "Commande sans modèle et commande à modèle restreint", $e$-STA, vol. 5, 2008 (soon online http://hal.inria.fr/).

[9] M. Fliess, C. Join, H. Sira-Ramírez, "Non-linear estimation is easy", Int. J. Modelling Identification Control, vol. 3, 2008 (online http://hal.inria.fr/inria-00158855/en/).

[10] Y. Fukada, "Slip-Angle Estimation for Vehicle Stability Control", Vehicle System Dynamics, vol. 32, p. 375-388, 1999.

[11] A. Hac, M.D. Simpson, "Estimation of vehicle side slip angle and yaw rate", Proc. SAE World Congress, Detroit, 2000.

[12] H.F. Grip, L. Imsland, T.A. Johansen, T. I. Fossen, J. C. Kalkkuhl, A. Suissa, "Nonlinear vehicle side-slip estimation with friction adaptation", Automatica, vol. 44, pp. 611-622, 2008.

[13] U. Kiencke, L. Nielsen, Automotive Control Systems for Engine, Driveline and Vehicle, Springer, 2005.

[14] M. Mboup, C. Join, M. Fliess, "A revised look at numerical differentiation with an application to nonlinear feedback control", Proc. 15 $5^{\text {th }}$ Medit. Conf. Control Automat., Athens, 2007 (online http://hal.inria.fr/inria-00142588/en/).

[15] J. Matusko, I. Petrovic, N. Peric, "Neural Network based tire/road friction force estimation", Engineering Applic. Artif. Intelligence, vol. 21, 2008.

[16] R.T. O’Brien Jr, K. Kiriakidis, "A comparison of $H_{\infty}$ with Kalman Filtering in vehicle state and parameter identification", Proc. Amer. Control Conf., Minneapolis, 2006.

[17] H. Pacejka, E. Baker, "The magic formula tyre mode 1 ", $1^{\text {st }}$ Internat. Coll. Tyre Models Vehicle System Analysis, pp. 1-18, 1991.

[18] A. Rahbi, N.K. M'Sirdi, N. Zbiri, Y. Delanne, "Vehicle-road interaction modelling for estimation of contact forces", Vehicle System Dynamics, vol. 43, pp. 403-411, 2005

[19] R. Rajamani, Vehicle Dynamics And Control, Springer, 2005.

[20] L. Ray, "Nonlinear tire force estimation and road friction identification: simulation and experiments", Automatica, vol. 33, pp. 1819-1833, 1997.

[21] B. Samadi, R. Kazemi K. Y. Nikravesh, M. Kabganian, "Real-Time estimation of vehicle state and tire-road friction forces", American Control Conf., Arlington, pp. 3318-3323, 2001.

[22] T. Shim, D. Margolis, "Model-Based Road Friction Estimation", Vehicle System Dynamics, Vol. 41, pp. 249-276, 2004.

[23] J. Stéphant, A. Charara, D. Meizel, "Evaluation of sliding mode observer for vehicle sideslip angle", Proc. $16^{\text {th }}$ IFAC World Congress, Prague, 2005.

[24] A.Y. Ungoren, H. Peng, H.E. Tseng "A study on lateral speed estimation methods", Int. J. Vehicle Autonomous Systems, Vol. 2, 2004.

[25] A. von Vietinghoff, M. Hiemer, U. Kiencke, "Nonlinear observer design for lateral vehicle dynamics", Proc. $16^{\text {th }}$ IFAC World Congress, Prague, 2005.

[26] J. Villagra, Conception optimisée de lois de commande et de paramètres pour les organes de liaison au sol, Thèse, École des Mines de Paris, 2006.

[27] K. Yosida, Operational Calculus: A Theory of Hyperfunctions, Springer, 1984 (translated from the Japanese). 